\title{
The Use of Polynomial Deflection Function in The Analysis of Thick Plates using Higher Order Shear Deformation Theory
}

\author{
Ignatius Chigozie Onyechere ${ }^{1 *}$, Owus Matthias Ibearugbulem ${ }^{2}$, Uchechukwu Collins Anya ${ }^{3}$, Kelechi Okechukwu Njoku ${ }^{4}$,
} Anthony Ugonna Igbojiaku ${ }^{5}$ and Ledum Suanu Gwarah ${ }^{6}$

$1,2,3,4,5$ Department of Civil Engineering, School of Engineering and Engineering Technology, Federal University of Technology, Owerri, Nigeria
${ }^{6}$ Department of Civil Engineering, Kenule Beeson Saro-Wiwa Polytechnic, Bori, Rivers State, Nigeria

DOI: $10.36348 /$ sjce.2020.v04i04.001

| Received: 28.05.2020 | Accepted: 05.06.2020 | Published: 18.06.2020

*Corresponding author: Ignatius Chigozie Onyechere

\section{Abstract}

In this work, polynomial deflection expression was used in the free-vibration investigation of thick rectangular plates with two different boundary conditions; one with its four edges clamped denoted with the acronym (CCCC) and another with two adjacent edges fixed and having simple supports at the other two adjacent edges denoted with the acronym (CCSS). The edge conditions of the various plates were fulfilled and used in generating the stiffness coefficients which were substituted into the analytical equation to obtain the non-dimensional frequency functions for the plates at different span-depth ratio (a/t) and aspect ratio (b/a). It was observed that at the same value of (b/a), there is an increase in the value of the non-dimensional frequency parameter as $(\mathrm{a} / \mathrm{t})$ increases. Also, at the same value of $(\mathrm{a} / \mathrm{t})$, there is a decrease in the value of the non-dimensional frequency parameter as (b/a) increases. The results obtained for the all edges clamped plate were compared with similar works by other researchers in the literature and were found to follow similar pattern and trend and were quite close.

Keywords: Boundary Conditions, Natural frequency, Span-depth ratio, polynomial expression, Thick Plates.

Copyright @ 2020: This is an open-access article distributed under the terms of the Creative Commons Attribution license which permits unrestricted use, distribution, and reproduction in any medium for non-commercial use (NonCommercial, or CC-BY-NC) provided the original author and source are credited.

\section{INTRODUCTION}

In recent times, thick plates find numerous applications in many engineering structures such as; bridges, jetties, oil platforms, heavy construction equipment, ships, Industrial buildings, etc. The increase in the application of thick plates in engineering structures has necessitated the need for efficient and faster methods of analysis of thick plates.

Many Scientists and Engineers have done much work on the analysis of thick and moderately thick plates. Thick plate theories differ from thin plate theories by the introduction of shear deformation effects on the displacements of the plate. The first published work on thick plate was carried out by Reisner in 1945 and was later refined by Mindlin in 1951 [1, 2]. The Reisner-Mindlin theory is called the First Order Shear Deformation Theory (FSDT). FSDT assumes that the line straight perpendicular to the midplane before deformation will keep on being straight after deformation but not necessarily perpendicular to the midplane [3]. This assumption however, is not certain, and thus, to correct for it's lapses, a shear correction factor is introduced into the analysis [4]. Nevertheless, the determination of this correction factor posses a great deal of challenges and the results obtained using FSDT in analysis of thick plates have high percentage errors, thus, the need to improve the FSDT [5]. Higher Order Shear Deformation Theories (HSDT) is an improvement on FSDT and thus, does not require shear correction factor and provides zero shear stress condition on the top and bottom of the plaes [5].

Many researchers have developed HSDT and applied same in the analysis of thick plates. [6] used a higher order shear deformation theory with eight unknown variables to investigate the vibration and buckling analysis of thick plates [7] applied trigonometric displacement functions and Navier's solution in the analysis of thick plates. Timoshenko beam expressions was developed and used by [8] to investigate the free-vibration of moderately thick plates [9]. Used two-eigenfunctions to study free-vibration of thick plates. Polynomial deflection expression was used by [10] to study free-vibration of rectangular thick plates with simple supports at all the four edges [11] developed exact displacement expressions for analysis of thick plates. Buckling analysis of thick plates with simple supports at all the four edges using orthogonal 
polynomial displacement functions were investigated by $[12,13]$. studied the analysis of non-prismatic columns using polynomial expressions as displacement functions while [14] studied bending properties of thick plates using polynomial expressions to represent the displacements. Most of the previous researchers on structural plates used either trigonometric or exponential expressions as their deflection function [15].
Present study used a polynomial expression to represent the deflection to make the analysis quick and easy. A simple linear equation developed from a higher order shear deformation theory in the works of [10] was applied in free-vibration analysis of rectangular thick plates with all edges clamped and one with two adjacent edges clamped and the other two adjacent edges having simple supports.

\section{ANALYTICAL EQUATION}

The analytical equation used in the present study was derived in [10] and presented here as;

$S_{11}+S_{12} \cdot\left[\frac{-S_{23} \cdot S_{31}+S_{33} \cdot S_{21}}{S_{32}{ }^{2}-S_{33} \cdot S_{22}}\right]+S_{13} \cdot\left[\frac{-S_{23} \cdot S_{21}+S_{22} \cdot S_{31}}{S_{32}{ }^{2}-S_{33} \cdot S_{22}}\right]=\frac{m a^{4} \lambda^{2}}{D}=\Lambda^{2}$

Where;

$S_{i j}=L_{i j} * 1 / T_{6}$

$L_{11}=H_{1}\left(T_{1}+\frac{2 T_{2}}{P^{2}}+\frac{T_{3}}{P^{4}}\right), L_{12}=-H_{2}\left(T_{1}+\frac{T_{2}}{P^{2}}\right), L_{13}=-H_{2}\left(\frac{T_{2}}{P^{2}}+\frac{T_{3}}{P^{4}}\right), L_{21}=L_{12}, L_{22}=T_{1} H_{3}+\left(\frac{1-\mu}{2 P^{2}}\right) T_{2} H_{3}+$

$\left(\frac{1-\mu}{2}\right) \propto^{2} K_{4} H_{4}, L_{23}=\left(\frac{1+\mu}{2 P^{2}}\right) K_{2} H_{3}, L_{31}=L_{13}, L_{32}=L_{23}, L_{33}=\left(\frac{1-\mu}{2 P^{2}}\right) T_{2} H_{3}+\frac{T_{3}}{P^{4}} H_{3}+\left(\frac{1-\mu}{2 P^{2}}\right) \propto^{2} T_{5} H_{4}$

$T_{1}=\int_{0}^{1} \int_{0}^{1}\left(\frac{\partial^{2} h}{\partial R^{2}}\right)^{2} \partial R \partial Q$

$T_{2}=\int_{0}^{1} \int_{0}^{1}\left(\frac{\partial^{2} h}{\partial R^{2}} \cdot \frac{\partial^{2} h}{\partial Q^{2}}\right) \partial R \partial Q$

$T_{3}=\int_{0}^{1} \int_{0}^{1}\left(\frac{\partial^{2} h}{\partial Q^{2}}\right)^{2} \partial R \partial Q$

$T_{4}=\int_{0}^{1} \int_{0}^{1}\left(\frac{\partial \mathrm{h}}{\partial \mathrm{R}}\right)^{2} \partial R \partial Q$

$T_{5}=\int_{0}^{1} \int_{0}^{1}\left(\frac{\partial \mathrm{h}}{\partial \mathrm{Q}}\right)^{2} \partial R \partial Q$

$T_{6}=\int_{0}^{1} \int_{0}^{1}(h)^{2} \partial R \partial Q$

$H_{1}=1, \quad H_{2}=0.79$,

$H_{3}=0.6325, \quad H_{4}=6.246$

$h$ is the shape function which depends on the boundary condition of the plate.

From Equation (1), we deduct that;

$\lambda=\frac{\Lambda}{a^{2}} \cdot \sqrt{\frac{D}{m}}$

$\lambda=\overline{\bar{\Lambda}} \cdot \frac{\pi^{2}}{a^{2}} \sqrt{\frac{D}{m}}$

Where;

$\overline{\bar{\Lambda}}=\frac{\Lambda}{\pi^{2}}$

$\lambda$ is the non - dimensional natural frequency / resonating frequency of the plates.

\section{BOUNDARY CONDITIONS}

Two basic edge conditions were treated in this study; simple support and fixed supports denoted with (S) and (C) respectively. A simple beam is made of two edges which could be any two of these edges. This produced two independent beams used in this study. They are shown in Fig.1 ( $a$ and $b$ ).

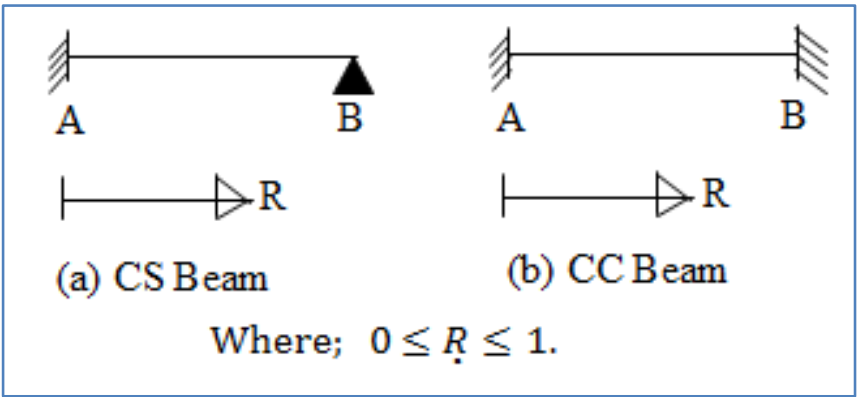

Fig-1: Edge conditions of the orthogonal beams 
Figs.1 ( $\mathrm{a}$ and $\mathrm{b}$ ) represent a beam fixed at one end and simply supported at the other end (CS beam) and a beam fixed at both edges (CC beam) respectively.

A plate is formed by grids of beams and these beams are arranged perpendicularly to each other. In combining them, the end conditions of the beam in x-direction (horizontal) are placed first before placing the end conditions of beam in y-direction (vertical) [2].

The deflection function ' $w$ ' for thick plates derived by [11] and presented here as.

$w=\left(a_{0}+a_{1} R+a_{2} R^{2}+a_{3} R^{3}+a_{4} R^{4}\right) *\left(b_{0}+b_{1} Q+b_{2} Q^{2}+b_{3} Q^{3}+b_{4} Q^{4}\right)$

Let;

$w=w_{x} * w_{y}$

Eq. (9) shows that a rectangular plate is a product of two independent orthogonal beams; one in $x(R)$-axis and the other in the $y(Q)$-axis. The deflection Equations for the two beams are given as

Eqs. (11a) and (11b) respectively

$w_{x}=\left(a_{0}+a_{1} R+a_{2} R^{2}+a_{3} R^{3}+a_{4} R^{4}\right)$

$w_{y}=\left(b_{0}+b_{1} Q+b_{2} Q^{2}+b_{3} Q^{3}+b_{4} Q^{4}\right)$

Differentiating Eqs. (11a) and (11b) with respect to $\mathrm{R}$ and $\mathrm{Q}$, yield

$\frac{\partial w_{x}}{\partial \mathrm{R}}=\left(a_{1}+a_{2} 2 \mathrm{R}+a_{3} 3 R^{2}+a_{4} 4 R^{3}\right)$
$\frac{\partial^{2} w_{x}}{\partial \mathrm{R}^{2}}=\left(2 a_{2}+a_{3} 6 \mathrm{R}+a_{4} 12 R^{2}\right)$
$\frac{\partial w_{y}}{\partial \mathrm{Q}}=\left(b_{1}+b_{2} 2 \mathrm{Q}+b_{3} 3 Q^{2}+b_{4} 4 Q^{3}\right)$
$\frac{\partial^{2} w_{y}}{\partial \mathrm{Q}^{2}}=\left(2 b_{2}+b_{3} 6 \mathrm{Q}+b_{4} 12 Q^{2}\right)$

\section{Boudary Conditions for C-C Beam}

For the beam fixed at both edges, the deflection and the slope end supports are zero.

(i) At $\mathrm{R}=0$, the deflection $w_{x}=0$

(ii) At $\mathrm{R}=1$, the deflection $w_{x}=0$

(iii) At $R=0$, Slope $=0$, i.e. $\frac{\partial w_{x}}{\partial R}=0$

(iv) $A t R=1$, Slope $=0$, i.e. $\frac{\partial w_{x}}{\partial R}=0$

Substituting Equations (12a)-(12b) and (12c)-(12d) into Eqs. (11a) and (11c) respectively, and solving appropriately yields;

$a_{0}=0 ; a_{1}=0 ; a_{2}=a_{4} ; a_{3}=-2 a_{4}$

Substituting Eq. (11) into Eq. (9a) yields;

$w_{x}=a_{4}\left(R^{2}-2 R^{3}+R^{4}\right)$

Similarly, repeating the same procedures for C-C boundary conditions in y $(\mathrm{Q})$ - direction, yield; $w_{y}=b_{4}\left(Q^{2}-2 Q^{3}+Q^{4}\right)$

\section{Boudary Conditions for C-S Beam}

For this beam, deflection and bending moment at the simple support are zero, while the deflection and slope at the fixed support are zero.

(i) At $\mathrm{R}=0$, the deflection $w_{x}=0$

(ii) At $R=0$, Slope $=0$, i.e. $\frac{\partial w_{x}}{\partial \mathrm{R}}=0$

(iii) At $\mathrm{R}=1$, the deflection $w_{x}=0$

(iv) At $R=1$, Bending Moment $=0$, i.e. $\frac{\partial^{2} w_{x}}{\partial R^{2}}=0$

Substituting Eqs. (15a), (15b) into Eqs. (11a), 11c), and Eqs. (15c), (15d) into Eqs. (11a) and ( $11 \mathrm{~d})$ and solving appropriately yields;

$a_{0}=0 ; a_{1}=0 ; a_{2}=1.5 a_{4} ; a_{3}=-2.5 a_{4}$

Substituting Eq. (16) into Eq. (11a) yields;

$w_{x}=a_{4}\left(1.5 R^{2}-2.5 R^{3}+R^{4}\right)$

(17a) 
The fractional equivalent of Eq. (17a) is;

$w_{x}=a_{4}\left(\frac{3}{2} R^{2}-\frac{5}{2} R^{3}+R^{4}\right)$

Similarly, repeating the same procedures for C-S boundary conditions in y (Q) - direction, yields;

$$
w_{y}=b_{4}\left(1.5 Q^{2}-2.5 Q^{3}+Q^{4}\right)
$$

The fractional equivalent of Eq. (17c) is;

$w_{x}=b_{4}\left(\frac{3}{2} Q^{2}-\frac{5}{2} Q^{3}+Q^{4}\right)$

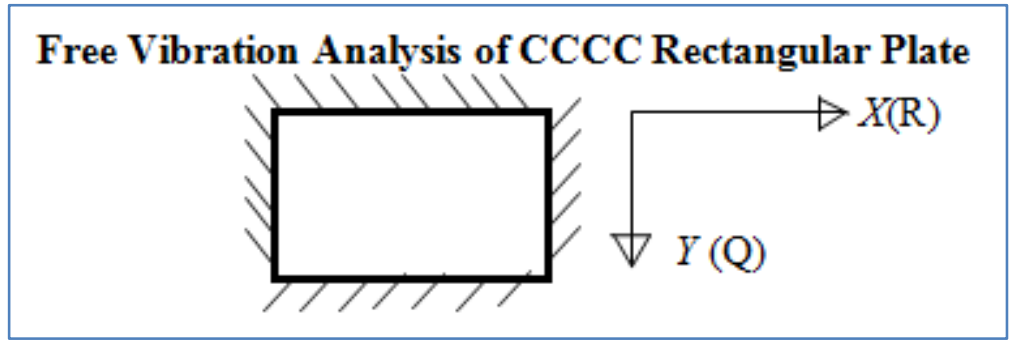

Fig-2: CCCC Rectangular Plate

The plate shown in Fig. 2, is a product of two perpendicularly placed C-C Beams, one in the horizontal direction $(\mathrm{x}(\mathrm{R})$ - axis) and the other in the vertical direction $(\mathrm{y}(\mathrm{Q})$ - axis. Thus, the expression for deflection is obtained by multiplying Eq. (14a) with Eq. (14b) which yields;

$w=a_{4}\left(R^{2}-2 R^{3}+R^{4}\right) \cdot b_{4}\left(Q^{2}-2 Q^{3}+Q^{4}\right)$

Upon simplification, yields;

$\mathrm{w}=A h=A\left(R^{2}-2 R^{3}+R^{4}\right) .\left(Q^{2}-2 Q^{3}+Q^{4}\right)$

Where;

$h=\left(R^{2}-2 R^{3}+R^{4}\right) \cdot\left(Q^{2}-2 Q^{3}+Q^{4}\right)$

$A=a_{4} b_{4}$

(20b)

' $A$ ' the amplitude and ' $h$ ' is the deflection expression for the CCCC thick plate.

Differentiating Equation (20a) with respect to $\mathrm{R}$ and $\mathrm{Q}$ yields;

$$
\begin{aligned}
& \frac{\partial h}{\partial R}=\left(2 R-6 R^{2}+4 R^{3}\right)\left(Q^{2}-2 Q^{3}+Q^{4}\right) \\
& \frac{\partial^{2} h}{\partial R^{2}}=\left(2-12 R+12 R^{2}\right)\left(Q^{2}-2 Q^{3}+Q^{4}\right) \\
& \frac{\partial h}{\partial Q}=\left(R^{2}-2 R^{3}+R^{4}\right)\left(2 Q-6 Q^{2}+4 Q^{3}\right) \\
& \frac{\partial^{2} h}{\partial Q^{2}}=\left(R^{2}-2 R^{3}+R^{4}\right)\left(2-12 Q-12 Q^{2}\right) \\
& \left(\frac{\partial^{2} h}{\partial R^{2}}\right)^{2}=\left(4-48 R+192 R^{2}-288 R^{3}+144 R^{4}\right)\left(Q^{4}-4 Q^{5}+6 Q^{6}-4 Q^{7}+Q^{8}\right)
\end{aligned}
$$

Recall from Eq. (4a);

$T_{1}=\int_{0}^{1} \int_{0}^{1}\left(\frac{d^{2} h}{d R^{2}}\right)^{2} \partial R \partial Q$

Upon substitution yields;

$T_{1}=\int_{0}^{1} \int_{0}^{1}\left(4-48 R+192 R^{2}-288 R^{3}+144 R^{4}\right)\left(Q^{4}-4 Q^{5}+6 Q^{6}-4 Q^{7}+Q^{8}\right) \partial R \partial Q(23 \mathrm{a})$

$T_{1}=\left(\frac{4}{1}-\frac{48}{2}+\frac{192}{3}-\frac{288}{4}+\frac{144}{5}\right) \cdot\left(\frac{1}{5}-\frac{4}{6}+\frac{6}{7}-\frac{4}{8}+\frac{1}{9}\right)=0.001270$

$T_{2}=\int_{0}^{1} \int_{0}^{1}\left(\frac{d^{2} h}{d R^{2}} \cdot \frac{d^{2} h}{d Q^{2}}\right) \partial R \partial Q$

$\left(\frac{d^{2} h}{d R^{2}} \cdot \frac{d^{2} h}{d Q^{2}}\right)=4\left[\left(R^{2}-8 R^{3}+19 R^{4}-18 R^{5}+6 R^{6}\right)\right] \cdot\left[\left(Q^{2}-8 Q^{3}+19 Q^{4}-18 Q^{5}+6 Q^{6}\right)\right.$

Integrating and evaluating Eq. (24b) yields;

$T_{2}=4 \int_{0}^{1} \int_{0}^{1}\left(R^{2}-8 R^{3}+19 R^{4}-18 R^{5}+6 R^{6}\right) \cdot\left(Q^{2}-8 Q^{3}+19 Q^{4}-18 Q^{5}+6 Q^{6}\right) \partial R \partial Q$ 


$$
\begin{aligned}
& T_{2}=4\left(\frac{1}{3}-\frac{8}{4}+\frac{19}{5}-\frac{18}{6}+\frac{6}{7}\right)^{2}=0.000363 \quad(24 \mathrm{~d}) \\
& T_{3}=\int_{0}^{1} \int_{0}^{1}\left(\frac{d^{2} h}{d Q^{2}}\right)^{2} \partial R \partial Q \\
& \left(\frac{\partial^{2} h}{\partial Q^{2}}\right)^{2}=\left(R^{4}-4 R^{5}+6 R^{6}-4 R^{7}+R^{8}\right) \cdot\left(4-48 Q+192 Q^{2}-288 Q^{3}+144 Q^{4}\right) \\
& T_{3}=\int_{0}^{1} \int_{0}^{1}\left(R^{4}-4 R^{5}+6 R^{6}-4 R^{7}+R^{8}\right) \cdot\left(4-48 Q+192 Q^{2}-288 Q^{3}+144 Q^{4}\right) \partial R \partial Q \\
& T_{3}=\left(\frac{1}{5}-\frac{4}{6}+\frac{6}{7}-\frac{4}{8}+\frac{1}{9}\right) \cdot\left(\frac{4}{1}-\frac{48}{2}+\frac{192}{3}-\frac{288}{4}+\frac{144}{5}\right)=0.001270 \\
& \left.T_{4}=\int_{0}^{1} \int_{0}^{1}\left(\frac{d h}{d R}\right)^{2} \partial R \partial Q \mathrm{c}\right) \\
& \left(\frac{\partial h}{\partial R}\right)^{2}=\left(4 R^{2}-24 R^{3}+52 R^{4}-48 R^{5}+16 R^{6}\right) \cdot\left(Q^{4}-4 Q^{5}+6 Q^{6}-4 Q^{7}+Q^{8}\right) \\
& T_{4}=\int_{0}^{1} \int_{0}^{1}\left(4 R^{2}-24 R^{3}+52 R^{4}-48 R^{5}+16 R^{6}\right) \cdot\left(Q^{4}-4 Q^{5}+6 Q^{6}-4 Q^{7}+Q^{8}\right) \partial R \partial Q
\end{aligned}
$$

Carrying out the integration yields;

$T_{4}=\left(\frac{4}{3}-\frac{24}{4}+\frac{52}{5}-\frac{48}{6}+\frac{16}{7}\right) \cdot\left(\frac{1}{5}-\frac{4}{6}+\frac{6}{7}-\frac{4}{8}+\frac{1}{9}\right)=0.0000302$

$T_{5}=\int_{0}^{1} \int_{0}^{1}\left(\frac{d h}{d Q}\right)^{2} \partial R \partial Q$

$\left(\frac{\partial h}{\partial Q}\right)^{2}=\left(R^{4}-4 R^{5}+6 R^{6}-4 R^{7}+R^{8}\right)\left(4 Q^{2}-24 Q^{3}+52 Q^{4}-48 Q^{5}+16 Q^{6}\right)$

$T_{5}=\int_{0}^{1} \int_{0}^{1}\left(R^{4}-4 R^{5}+6 R^{6}-4 R^{7}+R^{8}\right)\left(4 Q^{2}-24 Q^{3}+52 Q^{4}-48 Q^{5}+16 Q^{6}\right) \partial R \partial Q$

Upon Integration and evaluation yields;

$T_{5}=\left(\frac{1}{5}-\frac{4}{6}+\frac{6}{7}-\frac{4}{8}+\frac{1}{9}\right) \cdot\left(\frac{4}{3}-\frac{24}{4}+\frac{52}{5}-\frac{48}{6}+\frac{16}{7}\right)=0.0000302$

$T_{6}=\int_{0}^{1} \int_{0}^{1}(\mathrm{~h})^{2} \partial R \partial Q$

$(h)^{2}=\left(R^{4}-4 R^{5}+6 R^{6}-4 R^{7}+R^{8}\right) \cdot\left(Q^{4}-4 Q^{5}+6 Q^{6}-4 Q^{7}+Q^{8}\right)(32 \mathrm{~b})$

$T_{6}=\int_{0}^{1} \int_{0}^{1}\left(R^{4}-4 R^{5}+6 R^{6}-4 R^{7}+R^{8}\right) \cdot\left(Q^{4}-4 Q^{5}+6 Q^{6}-4 Q^{7}+Q^{8}\right) \partial R \partial Q$

Upon Integration and evaluation yields;

$T_{6}=\left(\frac{1}{5}-\frac{4}{6}+\frac{6}{7}-\frac{4}{8}+\frac{1}{9}\right) \cdot\left(\frac{1}{5}-\frac{4}{6}+\frac{6}{7}-\frac{4}{8}+\frac{1}{9}\right)=0.0000025$

Thus in summary, for CCCC thick plates we have;

$T_{1}=0.00127 ; T_{2}=0.000363 ; T_{3}=0.00127 ; T_{4}=0.0000302 ; T_{5}=0.0000302 ; T_{6}=0.0000025$

Substituting the Ti values and the Hi values of Eq. (5) into Eq. (3), the Lij values are obtained. Substituting the Lij values into Eq. (2), the Sij values are obtained. Substituting the Sij values into Eq. (1), $\Lambda^{2}$ is obtained. Substituting the $\Lambda^{2}$ values into Eq. (8), yields the values of the non-dimensional frequency function $\overline{\bar{\Lambda}}$ for the plate at various values of $(\mathrm{a} / \mathrm{t})$ and $(\mathrm{b} / \mathrm{a})$ as presented on Table 1.

\section{Free Vibration Analysis of CCSS Rectangular Plate}

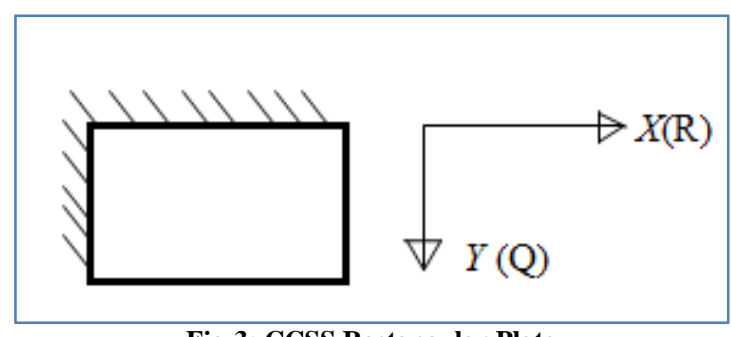

Fig-3: CCSS Rectangular Plate

The plate shown in Fig. 3, is a product of one C-S beam in the horizontal direction (x(R) - axis) and one C-S beam in the vertical direction $(\mathrm{y}(\mathrm{Q})$ - axis). Thus the deflection expression is obtained by multiplying Eq. (17ba) with Eq. (17d). This is shown as Eq. (34).

$w=a_{4}\left(1.5 R^{2}-2.5 R^{3}+R^{4}\right) \cdot b_{4}\left(1.5 Q^{2}-2.5 Q^{3}+Q^{4}\right)$

Eq. (34) can be rewritten as;

$w=A\left(1.5 R^{2}-2.5 R^{3}+R^{4}\right) \cdot\left(1.5 Q^{2}-2.5 Q^{3}+Q^{4}\right)=A h$ 
Where;

$h=\left(1.5 R^{2}-2.5 R^{3}+R^{4}\right) \cdot\left(1.5 Q^{2}-2.5 Q^{3}+Q^{4}\right)$

Where $A=a_{4} b_{4}$ is the amplitude and ' $\mathrm{h}$ ' is the deflection expression for CCSS thick plate.

Differentiating Eq. (37) with respect to $\mathrm{R}$ and $\mathrm{Q}$ yields;

$$
\begin{aligned}
& \frac{\partial h}{\partial R}=\left(3 R-\frac{15 R^{2}}{2}+4 R^{3}\right)\left(\frac{3 Q^{2}}{2}-\frac{5 Q^{3}}{2}+Q^{4}\right) \\
& \frac{\partial h}{\partial Q}=\left(\frac{3 R^{2}}{2}-\frac{5 R^{3}}{2}+R^{4}\right)\left(3 Q-\frac{15 Q^{2}}{2}+4 Q^{3}\right) \\
& \frac{\partial^{2} h}{\partial R^{2}}=\left(3-15 R+12 R^{2}\right)\left(\frac{3 Q^{2}}{2}-\frac{5 Q^{3}}{2}+Q^{4}\right) \\
& \frac{\partial^{2} h}{\partial Q^{2}}=\left(\frac{3 R^{2}}{2}-\frac{5 R^{3}}{2}+R^{4}\right)\left(3-15 Q+12 Q^{2}\right) \\
& T_{1}=\int_{0}^{1} \int_{0}^{1}\left(\frac{d^{2} h}{d R^{2}}\right)^{2} \partial R \partial Q
\end{aligned}
$$

$\left(\frac{\partial^{2} h}{\partial R^{2}}\right)^{2}=\left(9-90 R+297 R^{2}-360 R^{3}+144 R^{4}\right)\left(\frac{9 Q^{4}}{4}-\frac{30 Q^{5}}{4}+\frac{6 Q^{6}}{2}+\frac{25 Q^{6}}{4}-5 Q^{7}+Q^{8}\right)$

$T_{1}=\int_{0}^{1} \int_{0}^{1}\left(9-90 R+297 R^{2}-360 R^{3}+144 R^{4}\right)\left(\frac{9 Q^{4}}{4}-\frac{30 Q^{5}}{4}+\frac{6 Q^{6}}{2}+\frac{25 Q^{6}}{4}-5 Q^{7}+Q^{8}\right) \partial R \partial Q$

$T_{1}=\left(9-\frac{90}{2}+\frac{297}{3}-\frac{360}{4}+\frac{144}{5}\right) \cdot\left(\frac{9}{20}-\frac{30}{24}+\frac{6}{14}+\frac{25}{28}-\frac{5}{8}+\frac{1}{9}\right)=0.0135714$

$T_{2}=\int_{0}^{1} \int_{0}^{1}\left(\frac{d^{2} h}{d R^{2}} \cdot \frac{d^{2} h}{d Q^{2}}\right) \partial R \partial Q$

$\left(\frac{d^{2} h}{d R^{2}} \cdot \frac{d^{2} h}{d Q^{2}}\right)=9\left[\left(\frac{3 R^{2}}{2}-\frac{20 R^{3}}{2}+\frac{39 R^{4}}{2}-\frac{30 R^{5}}{2}+4 R^{6}\right)\right] \cdot\left[\left(\frac{3 Q^{2}}{2}-\frac{20 Q^{3}}{2}+\frac{39 Q^{4}}{2}-\frac{30 Q^{5}}{2}+4 Q^{6}\right)\right]$

$T_{2}=9 \int_{0}^{1} \int_{0}^{1}\left[\left(\frac{3 R^{2}}{2}-\frac{20 R^{3}}{2}+\frac{39 R^{4}}{2}-\frac{30 R^{5}}{2}+4 R^{6}\right)\right] \cdot\left[\left(\frac{3 Q^{2}}{2}-\frac{20 Q^{3}}{2}+\frac{39 Q^{4}}{2}-\frac{30 Q^{5}}{2}+4 Q^{6}\right)\right] \partial R \partial Q$

Upon integration and evaluation yields;

$T_{2}=9\left(\frac{3}{6}-\frac{20}{8}+\frac{39}{10}-\frac{30}{12}+\frac{4}{7}\right)\left(\frac{3}{6}-\frac{20}{8}+\frac{39}{10}-\frac{30}{12}+\frac{4}{7}\right)=0.00734694$

$T_{3}=\int_{0}^{1} \int_{0}^{1}\left(\frac{d^{2} h}{d Q^{2}}\right)^{2} \partial R \partial Q$

$\left(\frac{\partial^{2} h}{\partial Q^{2}}\right)^{2}=\left(\frac{9 R^{4}}{4}-\frac{30 R^{5}}{4}+\frac{6 R^{6}}{2}+\frac{25 R^{6}}{4}-5 R^{7}+R^{8}\right) \cdot\left(9-90 Q+297 Q^{2}-360 Q^{3}+144 Q^{4}\right)$

$T_{3}=\int_{0}^{1} \int_{0}^{1}\left(\frac{9 R^{4}}{4}-\frac{30 R^{5}}{4}+\frac{6 R^{6}}{2}+\frac{25 R^{6}}{4}-5 R^{7}+R^{8}\right) \cdot\left(9-90 Q+297 Q^{2}-360 Q^{3}+144 Q^{4}\right) \partial R \partial Q$

Upon integration and evaluation yields;

$T_{3}=\left(\frac{9}{20}-\frac{30}{24}+\frac{6}{14}+\frac{25}{28}-\frac{5}{8}+\frac{1}{9}\right) \cdot\left(\frac{9}{1}-\frac{90}{2}+\frac{297}{3}-\frac{360}{4}+\frac{144}{5}\right)=0.013571428$

$T_{4}=\int_{0}^{1} \int_{0}^{1}\left(\frac{d h}{d R}\right)^{2} \partial R \partial Q$

$\left(\frac{\partial h}{\partial R}\right)^{2}=\left(9 R^{2}-45 R^{3}+\frac{321 R^{4}}{4}-60 R^{5}+16 R^{6}\right) \cdot\left(\frac{9 Q^{4}}{4}-\frac{15 Q^{5}}{2}+\frac{37 Q^{6}}{4}-5 Q^{7}+Q^{8}\right)$

$T_{4}=\int_{0}^{1} \int_{0}^{1}\left(9 R^{2}-45 R^{3}+\frac{321 R^{4}}{4}-60 R^{5}+16 R^{6}\right) \cdot\left(\frac{9 Q^{4}}{4}-\frac{15 Q^{5}}{2}+\frac{37 Q^{6}}{4}-5 Q^{7}+Q^{8}\right) \partial R \partial Q$

Upon integration and evaluation yields;

$T_{4}=\left(\frac{9}{3}-\frac{45}{4}+\frac{321}{20}-\frac{60}{6}+\frac{16}{7}\right) \cdot\left(\frac{9}{20}-\frac{15}{12}+\frac{37}{28}-\frac{5}{8}+\frac{1}{9}\right)=0.0006462585$

$T_{5}=\int_{0}^{1} \int_{0}^{1}\left(\frac{d h}{d Q}\right)^{2} \partial R \partial Q$

$\left(\frac{\partial h}{\partial Q}\right)^{2}=\left(\frac{9 R^{4}}{4}-\frac{30 R^{5}}{4}+\frac{6 R^{6}}{2}+\frac{25 R^{6}}{4}-5 R^{7}+R^{8}\right)\left(9 Q^{2}-45 Q^{3}+\frac{321 Q^{4}}{4}-60 Q^{5}+16 Q^{6}\right)$

$T_{5}=\int_{0}^{1} \int_{0}^{1}\left(\frac{9 R^{4}}{4}-\frac{30 R^{5}}{4}+\frac{6 R^{6}}{2}+\frac{25 R^{6}}{4}-5 R^{7}+R^{8}\right)\left(9 Q^{2}-45 Q^{3}+\frac{321 Q^{4}}{4}-60 Q^{5}+16 Q^{6}\right) \partial R \partial Q$

Integrating and Evaluating yields;

$T_{5}=\left(\frac{9}{20}-\frac{30}{24}+\frac{6}{14}+\frac{25}{28}-\frac{5}{8}+\frac{1}{9}\right) \cdot\left(\frac{9}{3}-\frac{45}{4}+\frac{321}{20}-\frac{60}{6}+\frac{16}{7}\right)=0.000646259$

$T_{6}=\int_{0}^{1} \int_{0}^{1}(\mathrm{~h})^{2} \partial R \partial Q$

$(h)^{2}=\left(\frac{9 R^{4}}{4}-\frac{15 R^{5}}{2}+\frac{37 R^{6}}{4}-5 R^{7}+R^{8}\right) \cdot\left(\frac{9 Q^{4}}{4}-\frac{15 Q^{5}}{2}+\frac{37 Q^{6}}{4}-5 Q^{7}+Q^{8}\right)$

$T_{6}=\int_{0}^{1} \int_{0}^{1}\left(\frac{9 R^{4}}{4}-\frac{15 R^{5}}{2}+\frac{37 R^{6}}{4}-5 R^{7}+R^{8}\right) \cdot\left(\frac{9 Q^{4}}{4}-\frac{15 Q^{5}}{2}+\frac{37 Q^{6}}{4}-5 Q^{7}+Q^{8}\right) \partial R \partial Q$ 
Upon integration and evaluation yields;

$T_{6}=\left(\frac{9}{20}-\frac{15}{12}+\frac{37}{28}-\frac{5}{8}+\frac{1}{9}\right) \cdot\left(\frac{9}{20}-\frac{15}{12}+\frac{37}{28}-\frac{5}{8}+\frac{1}{9}\right)=0.0000568468$

Thus in summary, for CCSS thick plates we have;

$T_{1}=0.0135714 ; T_{2}=0.00734694 ; T_{3}=0.013571428 ; T_{4}=0.000646259 ; T_{5}=0.000646259 ; T_{6}=$ 0.0000568468 .

Substituting the Ti values and the Hi values of Eq. (5) into Eq. (3), the Lij values are obtained. Substituting the Lij values into Eq. (2), the Sij values are obtained. Substituting the Sij values into Eq. (1), $\Lambda^{2}$ is obtained. Substituting the $\Lambda^{2}$ values into Eq. (8), yields the values of the non-dimensional frequency function $\overline{\bar{\Lambda}}$ for the plate at various values of $(\mathrm{a} / \mathrm{t})$ and $(\mathrm{b} / \mathrm{a})$ as presented on Table 2.

\section{RESULTS AND DISCUSSIONS}

Table-1: Non-dimensional natural frequencies of CCCC thick plate

\begin{tabular}{|c|c|c|c|c|c|c|c|c|}
\hline \multirow{3}{*}{$\alpha=\mathbf{a} / \mathbf{t}$} & $\begin{array}{l}b / a \\
=1.0\end{array}$ & $\begin{array}{l}b / a \\
=1.2\end{array}$ & $\begin{array}{l}b / a \\
=1.5\end{array}$ & $\begin{array}{l}b / a \\
=1.8\end{array}$ & $\begin{array}{l}b / a \\
=2.0\end{array}$ & $\begin{array}{l}b / a \\
=2.2\end{array}$ & $\begin{array}{l}b / a \\
=2.4\end{array}$ & $\begin{array}{l}b / a \\
=2.6\end{array}$ \\
\hline & \multicolumn{8}{|c|}{$\lambda=\overline{\bar{\Lambda}} \cdot \frac{\pi^{2}}{a^{2}} \sqrt{\frac{D}{m}}$} \\
\hline & \multicolumn{8}{|c|}{$\overline{\bar{\Lambda}}$} \\
\hline 2 & 1.6949 & 1.5285 & 1.3874 & 1.3105 & 1.2780 & 1.2546 & 1.2371 & 1.2239 \\
\hline 5 & 2.8841 & 2.5289 & 2.2526 & 2.1158 & 2.0614 & 2.0234 & 1.9961 & 1.9758 \\
\hline 8 & 3.2882 & 2.8470 & 2.5183 & 2.3611 & 2.2996 & 2.2570 & 2.2265 & 2.2038 \\
\hline 10 & 3.4090 & 2.9397 & 2.5950 & 2.4316 & 2.3680 & 2.3241 & 2.2926 & 2.2693 \\
\hline 13 & 3.5057 & 3.0132 & 2.6554 & 2.4872 & 2.4219 & 2.3769 & 2.3446 & 2.3207 \\
\hline 16 & 3.5566 & 3.0516 & 2.6869 & 2.5161 & 2.4499 & 2.4043 & 2.3716 & 2.3474 \\
\hline 18 & 3.5780 & 3.0676 & 2.7000 & 2.5281 & 2.4616 & 2.4157 & 2.3829 & 2.3586 \\
\hline 21 & 3.5997 & 3.0840 & 2.7134 & 2.5404 & 2.4735 & 2.4273 & 2.3943 & 2.3699 \\
\hline 24 & 3.6141 & 3.0947 & 2.7221 & 2.5484 & 2.4813 & 2.4350 & 2.4018 & 2.3773 \\
\hline 27 & 3.6240 & 3.1021 & 2.7282 & 2.5540 & 2.4866 & 2.4402 & 2.4070 & 2.3825 \\
\hline 30 & 3.6312 & 3.1075 & 2.7326 & 2.5580 & 2.4905 & 2.4440 & 2.4108 & 2.3862 \\
\hline 33 & 3.6365 & 3.1114 & 2.7358 & 2.5610 & 2.4934 & 2.4469 & 2.4135 & 2.3889 \\
\hline 36 & 3.6406 & 3.1145 & 2.7383 & 2.5632 & 2.4956 & 2.4490 & 2.4157 & 2.3910 \\
\hline 39 & 3.6437 & 3.1168 & 2.7402 & 2.5650 & 2.4973 & 2.4507 & 2.4173 & 2.3926 \\
\hline 42 & 3.6463 & 3.1187 & 2.7417 & 2.5664 & 2.4987 & 2.4520 & 2.4186 & 2.3939 \\
\hline
\end{tabular}

Table-2: Non-dimensional natural frequencies of CCSS thick plate

\begin{tabular}{|c|c|c|c|c|c|c|c|c|}
\hline \multirow{3}{*}{$\alpha=\mathrm{a} / \mathrm{t}$} & $\begin{array}{l}b / a \\
=1.0\end{array}$ & $\begin{array}{l}b / a \\
=1.2\end{array}$ & $\begin{array}{l}b / a \\
=1.5\end{array}$ & $\begin{array}{l}b / a \\
=1.8\end{array}$ & $\begin{array}{l}b / a \\
=2.0\end{array}$ & $\begin{array}{l}b / a \\
=2.2\end{array}$ & $\begin{array}{l}b / a \\
=2.4\end{array}$ & $\begin{array}{l}b / a \\
=2.6\end{array}$ \\
\hline & \multicolumn{8}{|c|}{$\lambda=\overline{\bar{\Lambda}} \cdot \frac{\pi^{2}}{a^{2}} \sqrt{\frac{D}{m}}$} \\
\hline & \multicolumn{8}{|c|}{$\overline{\bar{\Lambda}}$} \\
\hline 2 & 1.5247 & 1.3656 & 1.2300 & 1.1555 & 1.1236 & 1.1001 & 1.0825 & 1.0689 \\
\hline 5 & 2.3470 & 2.0373 & 1.7902 & 1.6621 & 1.6089 & 1.5706 & 1.5421 & 1.5204 \\
\hline 8 & 2.5662 & 2.2045 & 1.9228 & 1.7792 & 1.7200 & 1.6776 & 1.6462 & 1.6222 \\
\hline 10 & 2.6264 & 2.2494 & 1.9580 & 1.8100 & 1.7492 & 1.7057 & 1.6734 & 1.6489 \\
\hline 13 & 2.6729 & 2.2839 & 1.9847 & 1.8335 & 1.7714 & 1.7270 & 1.6941 & 1.6691 \\
\hline 16 & 2.6967 & 2.3015 & 1.9984 & 1.8454 & 1.7827 & 1.7378 & 1.7046 & 1.6793 \\
\hline 18 & 2.7066 & 2.3088 & 2.0040 & 1.8503 & 1.7873 & 1.7422 & 1.7089 & 1.6835 \\
\hline 21 & 2.7167 & 2.3161 & 2.0097 & 1.8553 & 1.7920 & 1.7467 & 1.7132 & 1.6878 \\
\hline 24 & 2.7232 & 2.3209 & 2.0134 & 1.8585 & 1.7951 & 1.7497 & 1.7161 & 1.6906 \\
\hline 27 & 2.7277 & 2.3242 & 2.0159 & 1.8607 & 1.7972 & 1.7517 & 1.7181 & 1.6925 \\
\hline 30 & 2.7310 & 2.3266 & 2.0178 & 1.8623 & 1.7987 & 1.7531 & 1.7195 & 1.6939 \\
\hline 33 & 2.7334 & 2.3284 & 2.0191 & 1.8635 & 1.7998 & 1.7542 & 1.7205 & 1.6949 \\
\hline 36 & 2.7352 & 2.3297 & 2.0202 & 1.8644 & 1.8006 & 1.7550 & 1.7213 & 1.6957 \\
\hline 39 & 2.7367 & 2.3308 & 2.0210 & 1.8651 & 1.8013 & 1.7557 & 1.7219 & 1.6963 \\
\hline 42 & 2.7378 & 2.3316 & 2.0216 & 1.8657 & 1.8018 & 1.7562 & 1.7224 & 1.6967 \\
\hline
\end{tabular}


Table-3: Results of Present Study Compared with the Results of [5] for CCCC thick Plates

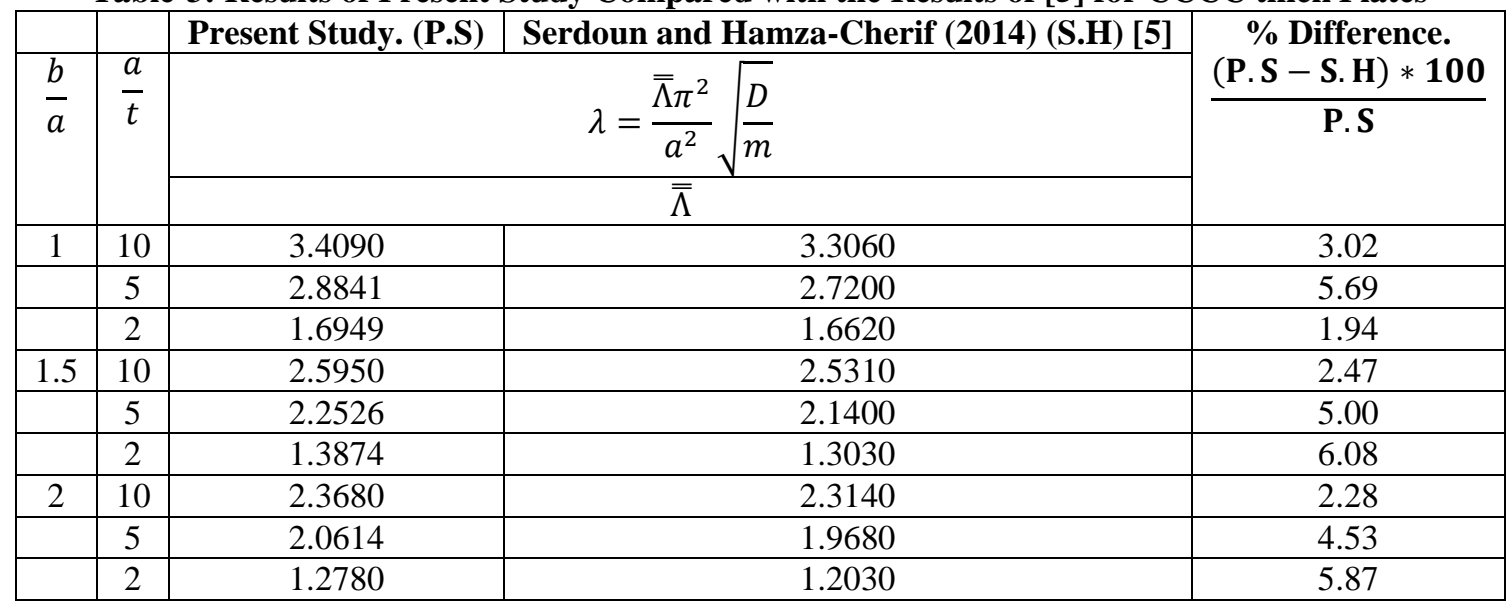

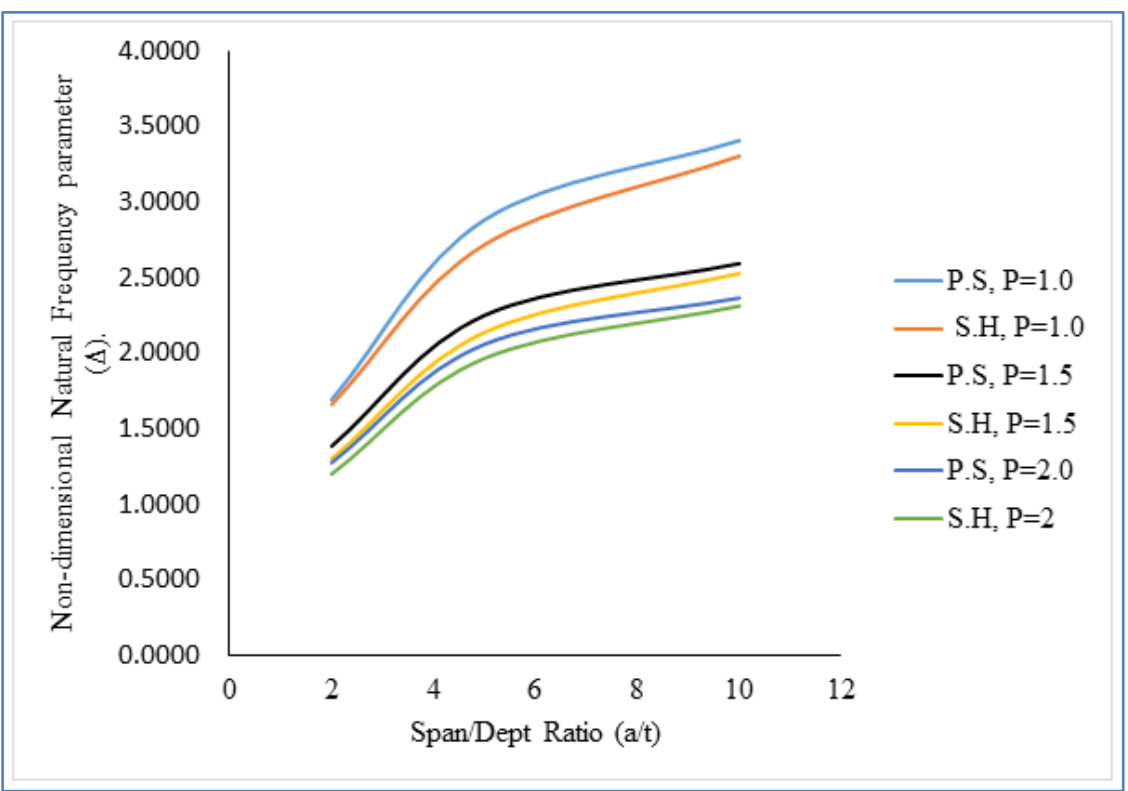

Fig-4: Present Study (P.S) result compared with the Results of Serdoun and Hamza-Cherif (2014) (S.H) [5], for vibration analysis of CCCC thick Plates at the various aspect ratio, $P=b / a$

From Tables 1 and 2, it is evident that at the same value of (b/a), there is an increase in the value of the non-dimensional frequency function $\overline{\bar{\Lambda}}$ as (a/t) increases with its lowest value occurring at $a / t=2$ and its highest value occurring at $\mathrm{a} / \mathrm{t}=42$ this implies that vibratory load has more impact on a plate as its spandepth ratio $(\mathrm{a} / \mathrm{t})$ increases. Also, at the same value of $(\mathrm{a} / \mathrm{t})$, there is a decrease in the value of the nondimensional frequency parameter as (b/a) increases with its lowest value occurring at $\mathrm{b} / \mathrm{a}=2.6$ and its highest value occurring at $\mathrm{b} / \mathrm{a}=1.0$, this implies that the strength capacity of the plate to resist vibration decreases as b/a increases with the square plate being the strongest plate. Table 3 and Fig. 4 shows that the results obtained for the all edges clamped plate upon comparison with the works of previous researchers were found to follow similar pattern and trend and were quite close.

\section{CONCLUSIONS}

The use of polynomial deflection expression in the free vibration analysis of thick rectangular plates with two different edge conditions; one with its four edges clamped denoted with the acronym (CCCC) and another with two adjacent edges fixed and the other two adjacent edges having simple supports denoted with the acronym (CCSS) was investigated using a simple linear equation derived in [10] based on higher order shear deformation theory.

Vibratory load has more impact on a plate as its span-depth ratio $(\mathrm{a} / \mathrm{t})$ increases. The strength capacity of the plate to resist vibration decreases as aspect ratio (b/a) increases with the square plate being the strongest plate.

The results from the present study follow similar pattern and trend and were quite close to the results of similar works in the literature. 
Through the use of the analytical equation, the non-dimensional natural frequency function of the plate could be easily determined at any value of span thickness ratio (a/t) and aspect ratio (b/a).

The use of polynomial expression as the deflection function makes the analysis less rigorous and faster as polynomial expressions are easily to manipulate mathematically.

\section{REFERENCES}

1. Senjanović, I., Vladimir, N., Cho, D. S., \& Choi, T. M. (2014, January). Vibration Analysis of Thick Plates: Analytical and Numerical Approaches. In ASME 2014 33rd International Conference on Ocean, Offshore and Arctic Engineering. American Society of Mechanical Engineers Digital Collection.

2. Onyechere, I. C. (2018). Stability and Vibration Analysis of Thick Plates using Orthogonal Polynomial Displacement Functions. Unpublished PhD Thesis Federal University of Technology, Owerri, Nigeria.

3. Sayyad, A. S., \& Ghugal, Y. M. (2012). Bending and free vibration analysis of thick isotropic plates by using exponential shear deformation theory. Applied and Computational mechanics, 6(1).

4. Sadrnejad, S. A., Daryan, A. S., \& Ziaei, M. (2009). Vibration equations of thick rectangular plates using mindlin plate theory. Journal of Computer Science, 5(11), 838.

5. Serdoun, S.M.N., \& Hamza-Cherif, S.M. (2014): Exact Characteristic Equations for Some of Classical Boundary Conditions of Vibrating Moderately Thick Rectangular Plates. American Scientific Research Journal for Engineering, Technology, and Sciences (ASRJETS), 9(1), 1-19.

6. Tran I. U., Tran M.U., Tran H.Q., \& Nguyen V.L. (2016). Vibration and Buckling Analysis of Functionally Graded Plates Using New EightUnknown Higher Order Shear Deformation Theory. Latin American Journal of Solids and Structures, 13(3), 456-477.
7. Sayyad, I.I., Chikalthankar S.B. and Nandedkar V.M. (2013). Bending and Free Vibration Analysis of Isotropic Thick Plate Using Refined Plate Theory. Bonfring International Journal of Industrial Engineering and Management Science, 3(2), 40 46.

8. Cheung, Y. K., \& Zhou, D. (2000). Vibrations of moderately thick rectangular plates in terms of a set of static Timoshenko beam functions. Computers \& Structures, 78(6), 757-768.

9. Xing, Y., \& Liu, B. (2009). Closed Form Solutions for Free Vibrations of Rectangular Mindlin Plates. Acta Mech Sin. 25, 689-698.

10. Onyechere, I. C., Ibearugbulem, O.M.M., Anya U. C., Anyaogu, L., \& Awodiji C.T.G. (2020). FreeVibration Study of Thick Rectangular Plates using Polynomial Displacement Functions, Saudi Journal of Engineering and Technology, 5(2), 73-80.

11. Ibearugbulem, O. M., Onyechere, I. C., Ezeh, J. C., \& Anya, U. C. (2019). Determination of Exact Displacement Functions for Rectangular Thick Plate Analysis, Futo Journal Series (FUTOJNLS), 5(1), 101-116.

12. Ezeh, J.C., Onyechere, I.C., Ibearugbulem, O.M, Anya, U. C., \& Anyaogu, L. (2018). Buckling Analysis of Thick Rectangular Flat SSSS Plates using Polynomial Displacement Functions, International Journal of Scientific \& Engineering Research, 9(9), 387-392.

13. Ibearugbulem O. M., Ezeh J. C., Ibarugbulem C. N., \& Onyechere I. C. (2020). Closed Form Stability Analysis of Solid Non-Prismatic Columns. International Journal of Innovative Science and Research Technology, 5(3), 105-113.

14. Ezeh, J.C., Ibearugbulem, O. M., Ettu, L. O., Gwarah, L. S., \& Onyechere, I. C. (2018). Application of Shear Deformation Theory for Analysis of CCCS and SSFS Rectangular Isotropic Thick Plates. IOSR Journal of Mechanical and Civil Engineering (IOSR-JMCE). 15(5), 33-42.

15. Ezeh, J. C., Ibearugbulem, O. M., \& Onyechere, C. I. (2013). Pure bending analysis of thin rectangular flat plates using ordinary finite difference method. International Journal of Emerging Technology and Advanced Engineering, 3(3), 2023. 